\title{
Buoyant Convection Computed in a Vorticity, Stream-Function Formulation
}

\author{
Ronald G. Rehm, * Howard R. Baum, $\dagger$ and P. Darcy Barnett* \\ National Bureau of Standards, Washington, DC 20234
}

September 2, 1981

\begin{abstract}
Model equations describing large scale buoyant convection in an enclosure are formulated with the vorticity and stream function as dependent variahles. The malhematical model, bascd on earlier work of the authors, is unique in two respects. First, it neglects viscous and thermal conductivity effects. Second the Inid is taken to be thermally expandable: large density variations are allowed while acoustic waves are filtered out. A volumetric heat source of specified spatial and lemporal variation drives the flow in a two-dimensional rectangular enclosure. An algorithm for solulion of the equations in this vorticily, stream-function formulation is presented. Results of computations using this algorithm are presented. Comparison of these results with those obtained carlier by the authors using a finite differenee code to inlegrate the primitive equations show excellent agreement. A method for periodically smoothing the computational results during a ealeulation, using Lanczos smoothing, is also presented. Conpulations with smoothing at different lime intervals are presented and diseussed.
\end{abstract}

Key words: buoyant convection; finite differenee eompulations; fire-enclosure; nuid flow; Lanezos smoothing; partial differential equations; stream Innclion; vorticity.

\section{Introduction}

Over the past few years, the National Bureau of Standards has sponsored a joint researeh projeet between the Center for Fire Research and the Center for Applied Mathematics to develop, starting from basic conservation laws, a mathematical model of fire development within a room. Large scale convection is an essential component of such a model because this fluid motion governs the smoke and hot gas transport within a room and also supplies fresh oxygen to the fucl to sustain eombustion. Therefore, development of a mathematical model of buoyant convection was begun as a first step toward a more complete room-fire model, which would include effects of combustion chemistry, radiation, and smoke dynamics. The mathematical model for convection, the partial differential equations, and boundary conditions, are derived in reference [1]. ${ }^{1}$

As noted in carlier papers $[1,2]$ the mathematical model is unique in two respects. First, it is assumed that viscous and thermal conductivity effects are negligible. Second, the fluid has been taken to be thermally expandable so that large temperature and density variations can be taken into account, while acoustic waves have been filtered out to reduce computational time.

The modcl equations were integrated for density, pressure, and velocity components by finite difference techniques; the algorithm is presented in detail in reference [2]. The algorithm has been verified by comparison with solutions to the equations in special cases obtained by analytical and independent numcrical means; the verification is described in references [3] and [4] and in the present study.

In section 2 the model equations are recast into a form such that the dependent variables are density, pressurc, vorticity, velocity potential, and stream function. This formulation, the so-called vorticity, streamfunction formulation, is an alternate one to that described in reference [2], which we call a primitive-variables formulation. An algorithm for integration of the equations in a vorticity, stream-function formulation is also presented in this section.

* Center for Applied Mathemalics, National Engineering Laboratory.

† Center for Fire Research, National Engineering Laboratory. 
Results from the two algorithms are compared: the results are in such good agreement that the difference cannot be seen on plots of the dependent variables. This comparison represents a final check on the validity of the integration algorithms and their computer code implementations. Therefore, we believe that the solutions obtained by these computations are excellent approximate solutions to the model partial differential equations presented in reference [1].

The model has been developed for two-dimensional, time-dependent fires evolving in a room (rectangular enclosure). The fire has been modeled as a volumetric heat source of specified spatial extent and temporal behavior. In section 3 density or temperature (which are inversely related at any specified time in this model), vorticity, pressure, and velocity potential plots at fixed times during the heating are shown for a sample computation.

It is well known that, because of the quadratic nonlinearity in convection, initially smooth flow fields become increasingly more fnrrowcd as time progrcsses; i.e., energy cascades from lower wavenumber modes to higher ones. The computational results display this behavior, and the flow field becomes more intricate with increasing time, the resolution of the grid providing the only limitation to the resolvable detail. However, such an accumulation of energy at a wavenumber inversely proportional to the grid size is both unphysical, and, if the computation is carried out long enough, disastrous. Local gradients of the dependent variables become much too large and the computation ultimately fails. Therefore, also shown in section 3 of this paper are preliminary considerations of smoothing or filtering of the computational results. Such smoothing acts analogously to viscosity and can be used to prolong the lifetime of the computation. A brief discussion of the effects of a particular type (Lanczos) of smoothing is presented, and resnlts obtaincd using this smoothing are shown.

\section{Formulation}

\subsection{Continuous equations}

In an earlier paper [1], the authors had derived a set of nonlinear equations describing very nonadiabatic buoyant flows of a nondissipative perfect gas. The magnitude and the spatial variation of the heat source (representing the exothermic reaction in a fre) were taken as known. The fluid and the fire source were assumed confined in a closed rectangular room with the center of the source along the floor. In contrast to reference [1], in this paper we consider only a completely enclosed room (no leaks), and when difference equations are introduced, we confine attention to the two dimensional evolution of the flow.

In this section, the equations derived in reference [1] for a two-dimensional configuration are rewritten so that vorticity and the stream function are primary variables, and the finitc difference methods used to solve the revised equations are presented.

Equations (11) of reference [1] are

$$
\begin{aligned}
& \frac{\partial \rho}{\partial t}+\frac{\partial}{\partial x_{i}}\left(\rho u_{i}\right)=0 \\
& \rho\left(\frac{\partial u_{i}}{\partial t}+u_{j} \frac{\partial u_{i}}{\partial x_{j}}\right)+\frac{\partial\left(p-p_{o}(t)\right)}{\partial x_{i}}-\rho k_{i} g=0 \\
& \rho C_{p}\left(\frac{\partial T}{\partial t}+u_{j} \frac{\partial T}{\partial x_{j}}\right)-\frac{d p_{o}}{d t}=Q\left(x_{i}, t\right) \\
& p_{o}(t)=\rho R T
\end{aligned}
$$

Here $\rho$ is density, $u_{i}$ the velocity in the $i^{i h}$ coordinate direction $(i=1,2,3), p$ is the pressure excess above the mean pressure $p_{o}(t)$ in the room, $T$ the tempcrature, $C_{p}$ the constant-pressure specific heat, $R$ the gas constant, $k_{i} g$ is the gravitational acccleration, and $Q\left(x_{i}, t\right)$ the speeified volumetric heat source. The spatially uniform mean pressure $p_{o}(t)$ depends only upon time and increases beeause of the heating within the room. It is determined in a completely enclosed room by the equation 


$$
\frac{d p_{o}}{d t}=\frac{\gamma-1}{V} \int_{V} Q\left(x_{i}, t\right) d V
$$

where $\gamma$ is the ratio of specific heats, $V$ is the volume of the room and the integration is performed over this entire volume.

We take the substantial derivative of the equation of state and use this with the energy equation to eliminate the temperature. The resulting equation deseribes the evolution of the density under heating

$$
\frac{\partial \rho}{\partial t}+u_{i} \frac{\partial \rho}{\partial x_{i}}=-\rho \frac{\partial u_{i}}{\partial x_{i}}=-\rho D\left(x_{j}, t\right)
$$

where

$$
D\left(x_{j}, t\right)=\frac{1}{\gamma p_{o}(t)}\left[(\gamma-1) Q\left(x_{j}, t\right)-\frac{d p_{o}}{d t}\right] .
$$

Equation (3) and the continuity equation identify $D\left(x_{i}, t\right)$ as the divergence

$$
\frac{\partial u_{i}}{\partial x_{i}}=D\left(x_{j}, t\right)
$$

Finally, as in reference [1], the equation for the spatially variable portion of the pressure is obtained by dividing the momentum equations by density and taking the divergence of these equations. The resulting equation is

$$
\frac{\partial}{\partial x_{i}}\left(\frac{1}{\rho} \frac{\partial p}{\partial x_{i}}\right)=-\left[\frac{\partial}{\partial x_{i}}\left(u_{j} \frac{\partial u_{i}}{\partial x_{j}}\right)+\frac{\partial D\left(x_{j}, t\right)}{\partial t}\right]
$$

The boundary eonditions on these equations are that velocity normal to any (impermeable) wall vanish.

$$
u_{i} n_{i}=0
$$

where $n_{i}$ are the normal components of a vector describing the boundary wall. From eqs (1) and these conditions, the appropriate boundary conditions on the pressure equation are obtained

$$
n_{i} \frac{\partial p}{\partial x_{i}}=\rho g n_{i} k_{i}
$$

In two dimensions (no dependence on $z$ ), these equations become

$$
\begin{aligned}
& \frac{\partial \rho}{\partial t}+u \frac{\partial \rho}{\partial x}+v \frac{\partial \rho}{\partial y}=-\rho D(x, y, t) \\
& \frac{\partial u}{\partial t}+1 / 2 \frac{\partial}{\partial x}\left(u^{2}+v^{2}\right)-v \omega=-\frac{1}{\rho} \frac{\partial p}{\partial x} \\
& \frac{\partial v}{\partial t}+1 / 2 \frac{\partial}{\partial y}\left(u^{2}+v^{2}\right)+u \omega=-\frac{1}{\rho} \frac{\partial p}{\partial y}-g \\
& \frac{\partial}{\partial x}\left(\frac{1}{\rho} \frac{\partial p}{\partial x}\right)+\frac{\partial}{\partial y}\left(\frac{1}{\rho} \frac{\partial p}{\partial y}\right)=-\frac{\partial D}{\partial t}+\frac{\partial}{\partial x}(v \omega)-\frac{\partial}{\partial y}(u \omega)-1 / 2 \nabla^{2}\left(u^{2}+v^{2}\right)
\end{aligned}
$$

where $x$ and $y$ are the horizontal and vertical coordinates with velocity components $u$ and $v$ respectively and 


$$
\omega=\frac{\partial v}{\partial x}-\frac{\partial u}{\partial y}
$$

is the only nonzero component of vorticity.

Equations (9) can be recast into a form in which the vorticity, the stream function and the velocity potential, together with density and pressure, are primary dependent variables. The velocity components can be written as

$$
\begin{aligned}
& u=\frac{\partial \phi}{\partial x}+\frac{\partial \psi}{\partial y} \\
& v=\frac{\partial \phi}{\partial y}-\frac{\partial \psi}{\partial x}
\end{aligned}
$$

where $\phi$ is the velocity potential and $\psi$ the stream funclion. Equations for $\phi$ and $\psi$ come from the divergence eq (5) and from the definition for vorticity (10)

$$
\begin{aligned}
& \nabla^{2} \phi=D(x, y, t) \\
& \nabla^{2} \psi=-\omega
\end{aligned}
$$

For these two elliptic equations, the stream function and the normal derivative of the potential are zero on the boundary:

$$
\frac{\partial \phi}{\partial n}=0 \quad \text { and } \quad \psi=0
$$

The equation describing the density evolution remains as it was in eqs (9), and an equation for the vortieity evolution comes from taking the curl of the two velocity equations in eqs (9).

$$
\frac{\partial \omega}{\partial t}+u \frac{\partial \omega}{\partial x}+v \frac{\partial \omega}{\partial y}+\omega D=-\frac{\partial}{\partial x}\left(\frac{1}{\rho} \frac{\partial p}{\partial y}\right)+\frac{\partial}{\partial y}\left(\frac{1}{\rho} \frac{\partial p}{\partial x}\right)
$$

In figure 1 a schematic diagram of a fire evolving in a room in two dimensions and a set of coordinate axes are shown. It is assumed that initially the enclosure is filled with quiescent, stratified fluid of density $\rho_{o}(y)$.

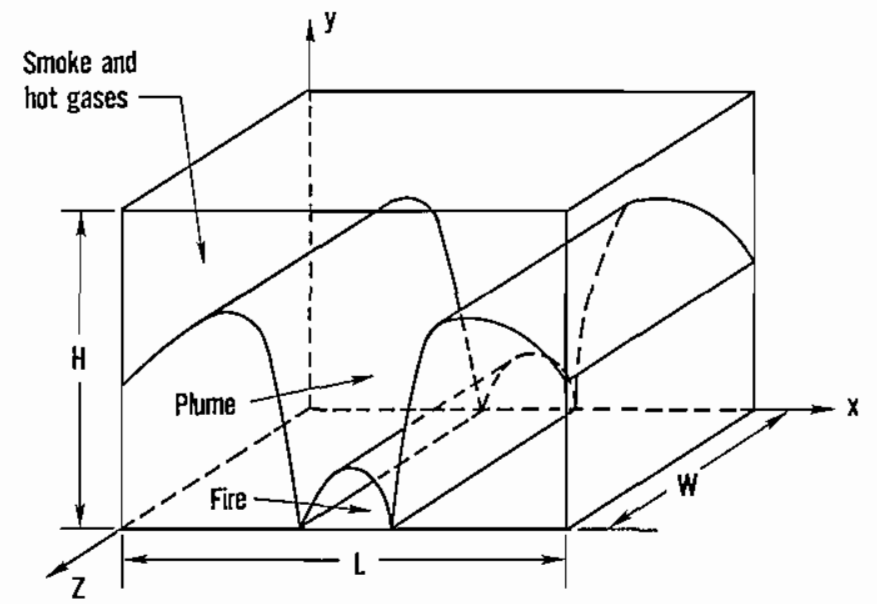

FIGURE 1. Schematic diagram of a two dimensional fire evolving in a room: it is assnmed that there is no dependence upon $z$ of any of the properties of the fire or of the induced flow field. The fire, loealized along the floor, has a plume of combustion products rising above it. The smoke and hot gases rise to the eeiling and fill the room from the top down. 
We define a density difference from ambient and a pressure difference as follows

$$
\begin{aligned}
& \widetilde{\rho}(x, y, t)=\rho(x, y, t)-\rho_{o}(y) \\
& \vec{p}(x, y, t)=p(x, y, t)-p_{o}(t)+g \int_{o}^{y} \rho_{o}\left(y^{\prime}\right) d y^{\prime}
\end{aligned}
$$

These differences $\bar{\rho}$ and $\tilde{p}$ need not be small compared with $\rho_{o}(y)$ and $p_{o}(t)$ respectively. Then the first and last of eqs (9) and eq (14) become

$$
\begin{aligned}
\frac{\partial \tilde{\rho}}{\partial t}+u \frac{\partial \tilde{\rho}}{\partial x}+v \frac{\partial \rho}{\partial y}+v \frac{d \rho_{o}}{d y}= & -\left(\rho_{o}(y)+\tilde{\rho}\right) D(x, y, t) \\
\frac{\partial \omega}{\partial t}+u \frac{\partial \omega}{\partial x}+v \frac{\partial \omega}{\partial y}+\omega D= & -\frac{\partial}{\partial x}\left[\left(1 /\left(\rho_{o}+\tilde{\rho}\right)\right)\left(\frac{\partial \tilde{p}}{\partial y}+g \tilde{\rho}\right)\right] \\
& +\frac{\partial}{\partial y}\left[\left(1 /\left(\rho_{o}+\tilde{\rho}\right)\right) \frac{\partial \tilde{p}}{\partial x}\right] \\
\frac{\partial}{\partial x}\left[\left(1 /\left(\rho_{o}+\tilde{\rho}\right)\right) \frac{\partial \tilde{p}}{\partial x}\right]+\frac{\partial}{\partial y}\left[\left(1 /\left(\rho_{o}+\tilde{\rho}\right)\right) \frac{\partial \tilde{p}}{\partial y}\right]= & -\frac{\partial}{\partial y}\left[g \tilde{\rho} /\left(\rho_{o}+\tilde{\rho}\right)\right] \\
& -\frac{\partial D}{\partial t}-(1 / 2) \nabla^{2}\left(u^{2}+v^{2}\right) \\
+ & \frac{\partial}{\partial x}(v \omega)-\frac{\partial}{\partial y}(u \omega)
\end{aligned}
$$

Equations (11), (12) and (15) constitute the complete set of differeutial equations for numerieal integration in the vorticity, stream function formulation. The boundary conditions are given by eqs (8), with $\tilde{p}$ and $\rho$ replacing $P$ and $\rho$, and by eqs (13).

Finally, we form dimensionless equations using the density $\rho_{o o} \equiv \rho_{o}(0)$, the height of the room $H$ as the length scale and the free fall time $(H / g)^{1 / 2}$ as the time scale. Then, denoting dimensionless quantities with a hat

$$
\begin{aligned}
\hat{\rho}=\hat{\rho} / \rho_{o o}, & \hat{p}=\tilde{p} /\left(\rho_{o o} g H\right), & \hat{\rho}_{o}=\rho / \rho_{o o} \\
\hat{\phi}=\frac{1}{H \sqrt{g H}} \phi, & \hat{\psi}=\frac{1}{H \sqrt{g H}} \psi, & \omega=\sqrt{\frac{H}{g} \omega} \\
\hat{u}=\frac{u}{\sqrt{g H}}, & \hat{v}=\frac{v}{\sqrt{g H}}, \quad \hat{x}=\frac{x}{H}, & \hat{y}=\frac{y}{H} \\
t=t / \sqrt{(H / g)} & &
\end{aligned}
$$

Equations (11), (12) and (15) remain exactly the same in dimensionless form with $g$ set equal to one. Subsequently, in this paper all quantities will be understood to be dimensionless, and the hat notation will be dropped. For the dimensionless coordinates, we note that $0 \leqslant x \leqslant 1 / A R$ and $0 \leqslant y \leqslant 1$ where $A R \equiv$ $H / L$.

\subsection{Discrete Equations}

\subsubsection{The Basic Algorithm}

In this section the finite difference equations and the boundary relations for the solution algorithm are presented. In figure $2 \mathrm{a}$, the two-dimensional rectangular enclosure in dimensionless variables is shown together with a sehematic representation of the spatial grids used for the finite difference scheme. The grid formed 


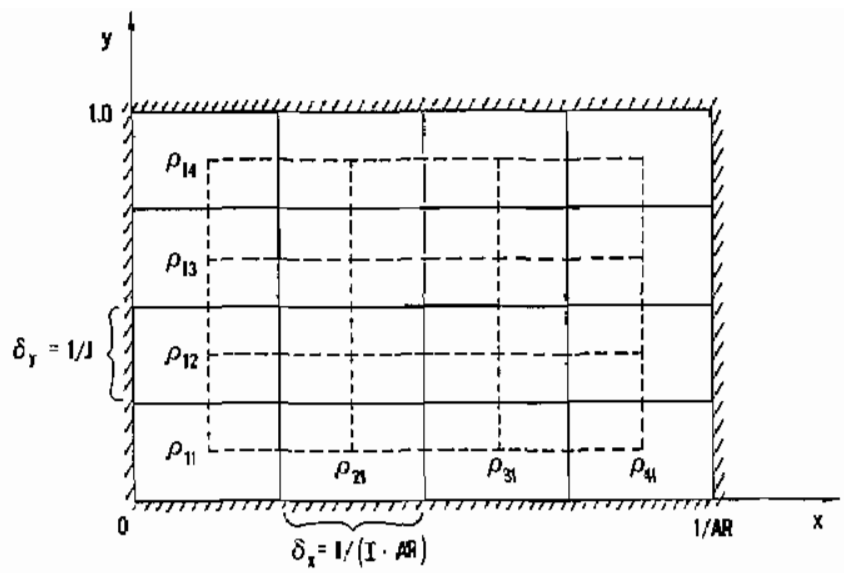

FIGCRE 2a. Rectangular enclosure in dinensionless variables $0 \leqslant x \leqslant \frac{1}{A R}$,

$0 \leqslant y \leqslant 1$. The mesh upon which the difference seheme is based is shown schemalically for $(I=J=4)$ as a grid of solid lines. The mesh of dashed limes joins the center points of the basie mesh cells and is the grid upon which the pressure computalion is performed.

from solid lines represents the basic mesh into which the enclosure is divided: in general there are $I$ mesh cells in the $x$-direction and $J$ mesh cells in the $y$-direetion.

Upon this basic mesh, the two components of the vector $(u, v)$ and single component of the vector vorticity $\omega=\frac{\partial v}{\partial x}-\frac{\partial u}{\partial y}$ are defined.

The second grid, formed by joining the center points of the basic grid cells and denoted by dashed lines, is that upon which scalar quantities such as density $\rho$ and pressure $p$ are defined. In figure $2 a$ the densities in the left-hand column of cells and in the bottom row of cells are shown to indicate how they are enumeraled for the numerical computation.

In figure $2 b$ a typical mesh cell is shown, illustrating where all of the dependent variables in the finite difference scheme are defined relative to the cell.

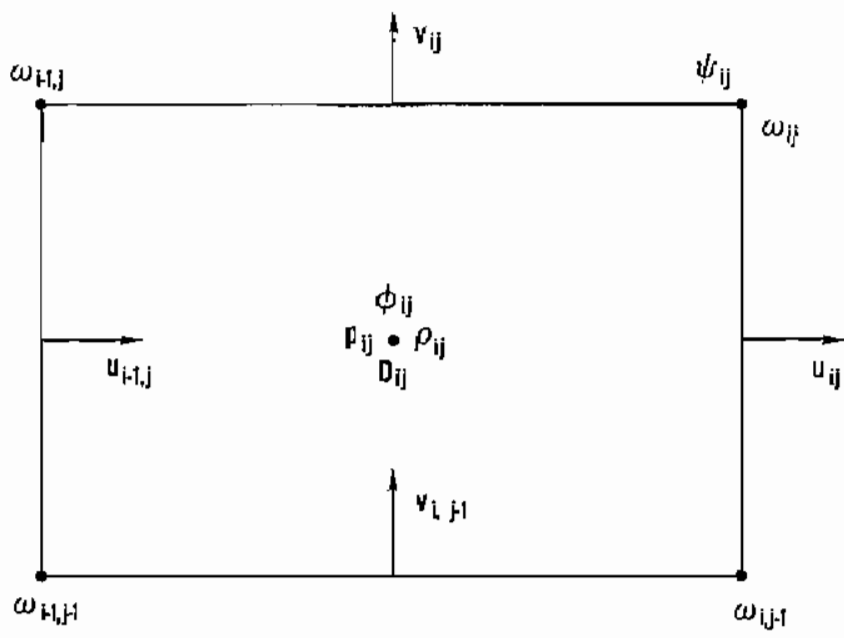

Ficune 2b. A typical mesh cell, with center localed at $x=(i-1 / 2) \delta x$ and $y=(j-1 / 2) \delta y$, illustrating where all dependent variables for the finile difference scheme are defined. 
The following diseretely evaluated funetions will denotc approximations to the eorresponding solutions to eqs (11), (12) and (15):

$$
\begin{aligned}
\phi_{i j}^{n} & \cong \phi((i-1 / 2) \delta x,(j-1 / 2) \delta y, n \delta t) \\
\psi_{i j}^{n} & \cong \psi(i \delta x, j \delta y, n \delta t) \\
u_{i j}^{n} & \cong u(i \delta x,(j-1 / 2) \delta y, n \delta t) \\
v_{i j}^{n} & \cong((i-1 / 2) \delta x, j \delta y, n \delta t) \\
\rho_{i j}^{n} & \cong \rho((i-1 / 2) \delta x,(j-1 / 2) \delta y, n \delta t) \\
p_{i j}^{n} & \cong p((i-1 / 2) \delta x,(j-1 / 2) \delta y, n \delta t) \\
D_{i j}^{n} & =D((i-1 / 2) \delta x,(j-1 / 2) \delta y, n \delta t), \\
\omega_{i j}^{n} & \cong \omega(i \delta x, j \delta y, n \delta t)
\end{aligned}
$$

where $\delta x=1 /(I \cdot A R)$ aud $\delta y=1 / J$ are the mesh cell sizes in the $x$ - and $y$-directions respectively and where $\delta t$ is the time-step size. Such a staggered grid is commonly used for multidimensional finite differenee integrations [5].

With this notation, the following set of finite difference equations was used to approximate eqs (11), (12) and (15) and boundary conditions (8) and (13):

For the first of eqs (15), $1 \leqslant i \leqslant I, 1 \leqslant j \leqslant J$ and $n \geqslant 2$,

$$
\begin{aligned}
{\sigma_{i j}^{n+1}}^{n+1}= & \frac{1}{1+(1 / 2) D_{i j}^{n} \delta t}\left\{\tilde{f}_{i j}^{n-1}\left(1-(1 / 2) D_{i j}^{n} \delta t\right)-2 \delta t\left(F_{\rho x_{i j}}^{n}+F_{\rho y_{j}}^{n}\right.\right. \\
& \left.\left.+(1 / 2) D_{i j}^{n} \rho_{o}(j)\right)\right\}
\end{aligned}
$$

where

$$
\begin{aligned}
& \hat{\rho}_{i j}^{n}=\rho_{i j}^{n}-\rho_{o}(j)=\text { the density difference from the initial density, } \\
& \rho_{o}(j)=\exp \left[-(j-1 / 2) \delta y / Y_{s}\right]=\text { the ambient, initial stratification, } \\
& Y_{s}=\text { the stratification length scale. }
\end{aligned}
$$

The flux terms $F_{\rho x i j}^{n}$ and $F_{\rho y_{i j}}^{n}$ for $1 \leqslant i \leqslant I, 1 \leqslant j \leqslant J$ are given by

$$
\begin{gathered}
F_{\rho x i j}^{n}=\left(\frac{2 \rho_{o}(j)+\rho_{i+1, j}^{n}+\rho_{i-1, j}^{n}}{4}\right)\left(\frac{u_{i j}^{n}-u_{i-1, j}^{n}}{\delta x}\right)+\left(\frac{\rho_{i+1, j}^{n}-\rho_{i-1, j}^{n}}{2 \delta x}\right)\left(\frac{u_{i j}^{n}+u_{i-1, j}^{n}}{2}\right) \\
F_{\rho 9 y, j}^{n}=\frac{\rho_{o}(j+1)+\rho_{o}(j-1)+\rho_{i, j+1}^{n}+\rho_{i, j-1}^{n}}{4}\left(\frac{v_{i j}^{n}-v_{i, j-1}^{n}}{\delta y}\right) \\
+\frac{\rho_{o}(j+1)-\rho_{o}(j-1)+\rho_{i, j+1}^{n}-\rho_{i, j-1}^{n}}{2 \delta y}\left(\frac{v_{i j}^{n}+v_{i, j-1}^{n}}{2}\right)
\end{gathered}
$$


For the second of eqs $(15), 1 \leqslant i \leqslant I, 1 \leqslant j \leqslant J$ and $n \geqslant 2$,

$$
\begin{aligned}
\omega_{i j}^{n+1}= & \omega_{i j}^{n-1}-2 \delta t\left\{\frac{1}{2 \delta x}\left(u_{\omega i+1, j}^{n} \omega_{i+1, j}^{n}-u_{\omega i-1, j}^{n} \omega_{i-1, j}^{n}\right)\right. \\
& \left.+\frac{1}{2 \delta y}\left(v_{\omega i, j+1}^{n} \omega_{i, j+1}^{n}-v_{\omega i, j-1}^{n} \omega_{i, j-1}^{n}\right)+G_{i j}^{n}\right\}
\end{aligned}
$$

where

$$
\begin{aligned}
v_{\omega i j}^{n}= & (1 / 2)\left(v_{i j}^{n}+v_{i+1, j}^{n}\right), \\
u_{\omega i j}^{n}= & (1 / 2)\left(u_{i, j+1}^{n}+u_{i j}^{n}\right), \\
G_{i j}^{n}= & \frac{2}{\delta x \delta y}\left[\frac{\tilde{p}_{i+1, j+1}^{n}-\tilde{\rho}_{i+1, j}^{n}+\left(\tilde{\rho}_{i+1, j+1}^{n}+\tilde{\rho}_{i+1, j}^{n}\right)(\delta y / 2)}{\rho_{o}(j+1)+\rho_{o}(j)+\hat{\rho}_{i+1, j+1}^{n}+\hat{\rho}_{i+1, j}^{n}}\right. \\
& -\frac{\tilde{p}_{i, j+1}^{n}-\tilde{p}_{i j}^{n}+\left(\tilde{\rho}_{i, j+1}^{n}-\hat{\rho}_{i j}^{n}\right)(\delta y / 2)}{\rho_{o}(\mathrm{j}+1)+\rho_{o}(j)+\hat{\rho}_{i, j+1}^{n}+\hat{\rho}_{i, j}^{n}} \\
& \left.-\frac{\tilde{p}_{i+1, j+1}^{n}-\tilde{p}_{i, j+1}^{n}}{2 \rho_{o}(j+1)+\tilde{\rho}_{i+1, j+1}^{n}+\hat{\rho}_{i, j+1}^{n}}+\frac{\tilde{p}_{i+1, j}^{n}-\tilde{p}_{i j}^{n}}{2 \rho_{o}(j)+\tilde{\rho}_{i+1, j}^{n}+\hat{\rho}_{i j}^{n}}\right]
\end{aligned}
$$

Equation (18) employs a second-order accurate temporal discretization which eliminates instability that would arise if leap frog had been applied. The first of eqs (15) has an undifferentiated term $\rho D(x, y, t)$ that is well known to lead to a eomputational instability for ordinary differential equations when leap frog is used. Reference [10] discusses the simple change in temporal differeneing used here to eliminate this instability.

Equation (21) uses a straightforward leapfrog temporal differeneing, and both eqs (18) and (21) are started by using the same spatial diseretization and an explicit, first-order time step.

At eaeh time step, after the vorticity has been updated, three elliptic equations must be solved, eqs (12a), (12b) and the last of eqs (15). Equations (12a) and (12b) are differenced using a standard five point star

$$
\begin{aligned}
& \frac{1}{\delta x^{2}}\left(\phi_{i+1, j}^{n}-2 \phi_{i j}^{n}+\phi_{i-1, j}^{n}\right)+\frac{1}{\delta y^{2}}\left(\phi_{i, j+1}^{n}-2 \phi_{i j}^{n}+\phi_{i, j-1}^{n}\right)=D_{i j}^{n} \\
& \frac{1}{\delta x^{2}}\left(\psi_{i+1, j}^{n}-2 \psi_{i j}^{n}+\psi_{i-1, j}^{n}\right)+\frac{1}{\delta y^{2}}\left(\psi_{i, j+1}^{n}-2 \psi_{i j}^{n}+\psi_{i, j-1}^{n}\right)=-\omega_{i j}^{n}
\end{aligned}
$$

and the boundary eonditions (13) are introdueed in the usual fashion. These equations have been solved using software routines from FISHPAK [6]: eq (24a) was solved using BLKTRI and more reeently using POISTG, while eq (24b) was solved using PWSCRT and more recently GENBUN. Routines BLKTRl and PWSCRT have limitations on the number of mesh points or unknowns which they can solve, whereas POISTG and GENBUN were produced more reeently and are free of sueh limitations. Most computations were performed with the former routines, but recently several computations have been performed using the latter.

The veloeities are then obtained from the potential and stream function by difference forms of eqs (11):

$$
\begin{aligned}
& u_{i j}^{n}=\frac{1}{\delta x}\left(\phi_{i+1, j}^{n}-\phi_{i j}^{n}\right)+\frac{1}{\delta y}\left(\psi_{i j}^{n}-\psi_{i, j-1}^{n}\right) \\
& v_{i j}^{n}=\frac{1}{\delta y}\left(\phi_{i, j+1}^{n}-\phi_{i j}^{n}\right)-\frac{1}{\delta x}\left(\psi_{i j}^{n}-\psi_{i-1, j}^{n}\right)
\end{aligned}
$$


For the third of eqs (15), for $1 \leqslant i \leqslant I$ and $1 \leqslant j \leqslant J$,

$$
\begin{aligned}
& \frac{2}{\delta x^{2}}\left[\frac{\tilde{p}_{i+1, j}^{n}-\tilde{p}_{i j}^{n}}{2 \rho_{o}(j)+\hat{\rho}_{i+1, j}^{n}+\hat{\rho}_{i j}^{n}}-\frac{\tilde{p}_{i j}^{n}-\tilde{p}_{i-1, j}^{n}}{2 \rho_{o}(j)+\hat{\rho}_{i j}^{n}+\hat{\rho}_{i-1, j}^{n}}\right] \\
& +\frac{2}{\delta y^{2}}\left[\frac{\tilde{p}_{i, j+1}^{n}-\tilde{p}_{i j}^{n}}{\rho_{o}(j+1)+\rho_{o}(j)+\tilde{\rho}_{i, j+1}^{n}+\hat{\rho}_{i j}^{n}}-\frac{\tilde{p}_{i j}^{n}-\tilde{p}_{i, j-1}^{n}}{\rho_{o}(j)+\rho_{o}(j-1)+\tilde{\rho}_{i j}^{n}+\hat{\rho}_{i, j-1}^{n}}\right] \\
& =-\frac{D_{i j}^{n+1}-D_{i j}^{n-1}}{2 \delta t}+\frac{F_{x i-1, j}^{n}-F_{x i j}^{n}}{\delta x}+\frac{F_{i j, j-1}^{n}-F_{r i j}^{n}}{\delta y} \\
& -\frac{1}{\delta y}\left[\frac{\hat{\rho}_{i, j+1}^{n}+\hat{\rho}_{i j}^{n}}{\rho_{o}(j+1)+\rho_{o}(j)+\hat{\rho}_{i, j+1}^{n}+\hat{\rho}_{i j}^{n}}-\frac{\hat{\rho}_{i j}^{n}+\hat{\rho}_{i, j-1}^{n}}{\rho_{o}(j)+\rho_{o}(j-1)+\hat{\rho}_{i j}^{n}+\hat{\rho}_{i, j-1}^{n}}\right]
\end{aligned}
$$

where the fluxes $F_{x i j}^{n}$ and $F_{y i j}^{n}$ are defined as follows:

for $1 \leqslant i \leqslant I-1,1 \leqslant j \leqslant J$

$$
F_{x i j}^{n}=\frac{1}{2 \delta x}\left[\left(q_{i+1, j}^{n}\right)^{2}-\left(q_{i j}^{n}\right)^{2}\right]-\frac{1}{2}\left(v_{\omega_{i j}^{n}}^{n} \omega_{i j}^{n}+v_{\omega_{i, j}-1}^{n} \omega_{i, j-1}^{n}\right)
$$

and for $1 \leqslant j \leqslant J-1,1 \leqslant i \leqslant I$

$$
F_{y i j}^{n}=\frac{1}{2 \delta y}\left[\left(q_{i, j+1}^{n}\right)^{2}-\left(q_{i j}^{n}\right)^{2}\right]+\frac{1}{2}\left(u_{\omega_{i j}}^{n} \omega_{i j}^{n}+u_{\omega_{i}-1, j}^{n} \omega_{i-1, j}^{n}\right)
$$

and where

$$
\begin{aligned}
\omega_{i j}^{n} & =\frac{v_{i+1, j}^{n}-v_{i j}^{n}}{\delta x}-\frac{u_{i, j+1}^{n}-u_{i j}^{n}}{\delta y} \\
v_{\omega_{i j}}^{n} & =\frac{1}{2}\left(v_{i j}^{n}+v_{i+1, j}^{n}\right), \quad u_{\omega_{i j}^{n}}^{n}=\frac{1}{2}\left(u_{i, j+1}^{n}+u_{i j}^{n}\right) \\
\left(q_{i j}^{n}\right)^{2} & =\left(\frac{u_{i j}^{n}+u_{i-1, j}^{n}}{2}\right)^{2}+\left(\frac{v_{i j}^{n}+v_{i, j-1}^{n}}{2}\right)^{2}
\end{aligned}
$$

Note that boundary conditions (7) on the normal velocities imply that $u_{0, j}=u_{I, j}=0$ for $1 \leqslant j \leqslant J$ and $v_{i, 0}=v_{i, J}=0$ for $1 \leqslant i \leqslant I$. These boundary conditions are applied formally in the expressions for the fluxes $F_{\alpha x_{i j}}^{n}, F_{\rho y_{i j}}^{n}, F_{x_{i j}}^{n}$ and $F_{y_{i j}}^{n}$ in mesh cells adjacent to boundaries. The boundary conditions (8) in discrete form become

$$
\begin{aligned}
& \tilde{p}_{0, j}^{n}=\tilde{p}_{1, j}^{n} \\
& \qquad \text { for } 1 \leqslant j \leqslant J \\
& \tilde{p}_{I, j}^{n}=\vec{p}_{I+1, j}^{n}
\end{aligned}
$$




$$
\begin{array}{ll}
\tilde{p}_{i, 1}^{n}-\tilde{p}_{i, 0}^{n}=\delta y\left(\tilde{p}_{i, 1}^{n}+\tilde{\rho}_{i, 0}^{n}\right) / 2 & \\
& \text { for } 1 \leqslant i \leqslant I \\
\tilde{p}_{i, J+1}^{n}-\tilde{p}_{i, J}^{n}=-\delta y\left(\tilde{p}_{i, J+1}^{n}+\tilde{\rho}_{i, J}^{n}\right) / 2 &
\end{array}
$$

We note that eqs (26) together with boundary conditions (28) constitute a singular linear algebraic system of equations. When eqs (26), with boundary conditions incorporated, are summed, the left hand side sums to zero, demonstrating that all of the equations are not linearly independent. Also, the last three terms on the right hand side sum to zero, producing the requirement that the double sum over $\left(D_{i j}^{n+1}-D_{i j}^{n-1}\right) / 2 \delta t$ must vanish. Examination of eq (30) below for $D_{i j}^{n}$ shows that it has been chosen so that its double sum oyer all mesh points vanishes. and that the eondition which must be satisfied to allow this choice produces eq (31) below for the mean pressure. Therefore, the singular linear algebraie system is seen to be consistent and thus to allow a solution. The solution is unique by noting that the double sum over all mesh points of $\bar{p}_{i j}^{n}$ must be zero.

At eaeh time step it is necessary to ealeulate the solution of the linear algebraic system for the pressure, eqs (26) with boundary conditions (28) incorporated. The method of solution must take into account nonuniqueness of the algebraic system. The solution method must also be able to solve large linear systems accurately, sinee there are $I J$ equations and cumulative errors from many time steps may destroy the computation. Finally, it is very important that the solution be obtained quiekly since the calculation is made at each time step, and hundreds of time steps must be taken.

The solution method we have adopted is a hybrid method which eombines an iterative algorithm, eonjugate gradients, with a fasi dircet Poisson solver. The eonjugate gradients algorithm provides an iterative technique for solving the linear algebraic system of equations. Details of the algorithm are presented in reference [7].

The heat source has been chosen to have the form

$$
\begin{aligned}
Q_{i j}^{n} & =\hat{Q}_{i j} f^{n} \\
\hat{Q}_{i j} & =\left(\frac{\beta}{\pi}\right)^{1 / 2} \lambda \exp \left[-\beta\left(x_{i}-x_{c}\right)^{2}-\lambda y_{j}\right] \\
x_{i} & =(i-1 / 2) \delta x, \quad y_{j}=(j-1 / 2) \delta y \\
f^{n} & =Q_{o} \tanh A t^{n} \\
t^{o} & =0, \quad t^{n}=\sum_{n^{\prime}=0}^{n-1} \delta t^{n^{\prime}}
\end{aligned}
$$

Henee, the diserete divergenee of the velocity field becomes

$$
\begin{aligned}
D_{i j}^{n} & =\frac{1}{\gamma p_{o}^{n}}\left[(\gamma-1) \hat{Q}_{i j}-K\right] f^{n} \\
K & =\frac{\gamma-1}{I J} \sum_{i=1}^{I} \sum_{i=1}^{J} \hat{Q}_{i j}
\end{aligned}
$$

and the mean background pressure is found from the difference equation

$$
p_{o}^{n+1}=p_{o}^{n-1}+K f^{n} 2 \delta t^{n}
$$

with $p_{o}^{0}=p_{o}^{1}=1$ since $f^{\circ}=0$. 
The linear stability of the algorithm is the only other eonsideration for diseussion. A linear stability analysis of eq (18) for the density shows that the time step $\delta t$ must satisfy the following condition for stability

$$
\delta t^{n} \leqslant \max _{\substack{1 \leqslant i \leqslant I \\ 1 \leqslant j \leqslant J}}\left\{\left(D_{i j}^{n}\right)^{2}+\left[\frac{\left|U_{i j}^{n}\right|}{\delta x}+\frac{\left|V_{i j}^{n}\right|}{\delta y}\right]^{2}\right\}^{-1 / 2}
$$

where

$$
\begin{gathered}
U_{i j}^{n} \equiv(1 / 2)\left(u_{i j}^{n}+u_{i-1, j}^{n}\right) \\
V_{i j}^{n} \equiv(1 / 2)\left(v_{i j}^{n}+v_{i, j-1}^{n}\right)
\end{gathered}
$$

When the stability eondition, eq (32), is not satisfied by a time step, the time step $\delta t^{11}$ is halved. Then the time-marching algorithm is restarted using the last time-level values as initial eonditions. A first-order time step is taken and then leap-frog is resumed.

A linear stability analysis of the difference equation for the vorticity, eq (21), yields exactly the same form for the stability criterion as that found above for the density equation. Reference to figure $2 b$ shows that the density and vorticity are evaluated at different points in the mesh, however, and therefore, the divergence $D$ and the velocity components $U$ and $V$ are to be evaluated at different points than those used in eq (32). To account for the difference in the stability criterion implied above, in all calculations performed using the algorithm deseribed above, the time step was chosen to be less than or equal to 0.8 the maximum value found for the right hand side of eq (32).

\subsubsection{Lanezos Smoothing}

The nonlincar nature of the equations of fluid dynamics implies that initially smooth data will, in general, produee flow fields with fine structure. Since the results presented are for finite difference computations, the resolution of the flow field is limited by the grid size used to perform the computation: structures of a size comparable to a few grid cells can be resolved, whereas smaller structures may represent artifacts of the calculations. In addition, in the computations it has been found that the calculation will eventually fail because of the intricate detail (and sharp gradicnts this detail represents) if the number of time steps becomes large cnough.

It is for thesc reasons that various methods of smoothing data generated by the computations have been cxamined. In the method presented here, the computation is stopped periodically, with a period specified as input to the computation, and the data are smoothcd spatially. The computation is restarted using the smoothed data as initial conditions, the results not being allowed to develop the intricate detail it might otherwise develop. The method used to smooth the data is a variation of one suggested by Lanczos in reference [8]. Using this method with a relatively long smoothing period, computations have been extended indefinitely.

The smoothing used here is that proposed by Lanczos, but is modified slightly for our purposes. In reference [8], the smoothed data is obtained from the value of the data at each point by adding a specified multiple of the fourth difference at that point. The change in value between the smoothed and unsmoothed data then is of order $h^{4}$ where $h \cong 1 / J$ and $J$ is the number of mesh points in one direction in space.

Since the computational scheme described here is only second order accurate in the spatial mesh size, $0\left(h^{2}\right)$, a less refined smoothing was used. The smoothing is accomplished by adding a specific multiple of the second-order difference at the point to the value of the datum at that point and is $0\left(h^{2}\right)$. When the method is generalized to two dimensions, it becomes equivalent to adding one fifth of the finite difference, five-point Laplacian to the value of the daturn at each point to obtain the smoothed datum. (This is also equivalent to replacing the value at a point by the average of its value and the values of its four nearest neighbors.)

Therefore, after a specified number of time steps $m$, the density and vorticity data at time level $m$ are changed according to the following prescription: 


$$
\begin{aligned}
& \rho_{i j}^{m} \leftarrow \rho_{i j}^{m}+\frac{1}{5}\left(\rho_{i+1, j}^{m}+\rho_{i-1, j}^{m}+\rho_{i, j+1}^{m}+\rho_{i, j-1}^{m}-4 \rho_{i j}^{m}\right) \\
& \omega_{i j}^{m} \leftarrow \omega_{i j}^{m}+\frac{1}{5}\left(\omega_{i+1, j}^{m}+\omega_{i-1, j}^{m}+\omega_{i, j+1}^{m}+\omega_{i, j-1}^{m}-4 \omega_{i j}^{m}\right)
\end{aligned}
$$

At the boundaries, the following rules are used for the smoothing. For cells adjacent to boundaries, the density is assumed to have the same value in a ficticious cell outside the boundary as its value in the cell under consideration. (This is the difference equivalent of saying that the normal derivative of the density at a boundary is zero.) The vorticity is taken to be zero on the boundary, and represents a free slip boundary condition,

A rough estimate of an equivalent Reynolds number or Grashof number introduced by smoothing can be made by the following argument. The effect of viscosity in the vorticity equation in the Boussinesq approximation arises, when the equations are made dimensionless in an appropriate way, as $\frac{1}{R e} \nabla^{2} \omega$, where $R e$ is the Reynolds number, $\nabla^{2}$ is the Laplacian and $\omega$ is the vorticity. The average effect per time step of Lanczos smoothing in the vorticity equation can also be represented as $\frac{c h^{2}}{m \delta} \nabla^{2} \omega$ where $c$ is a constant of order unity, $\delta$ is the time step, $h$ is the mesh spacing, $m$ is the number of steps between smoothings and $\nabla^{2}$ is the discretized, five-point representation of the Laplacian. Equating the coefficients of the Taplacian operators provides an estimate of the Reynolds number introduced by smoothing:

$$
R e=\frac{\delta}{c} \frac{m}{h^{2}}=\frac{\delta}{c} m I \cdot J
$$

where $I$ and $J$ are the number of mesh points in each direction. Taking $c=1, \delta=0.1, m=40, I \cdot J \cong$ 1000 , then $R e \cong 4 \times 10^{3}$, and noting that the Grashof number $G r$ is approximately the Reynolds number squared, $G r \cong 1.6 \times 10^{7}$.

\section{Computational Results}

As discussed in the Introduction, the vorticity, stream-function algorithm and a code implementing this algorithm were developed as a method for solving the partial differential cquations derived in reference [1]. The other method for solving these equations, a finite difference method for directly integrating the equations of motion in primitive variables (density, pressure and the two components of velocity) was described in reference [2]. Reference [3] describes comparisons of the results computed using the primitive variable algorithm with analytical results obtained in special cases. These comparisons were performed to test the algorithm and the computer-code implemcntation. Final comparisons were madc between results computed using the primitive variables code and those eomputed using the vorticity, stream-function code. Agreement between results was found to about five significant figures after a few time steps and to between three and four significant figures after hundreds of time steps. The diserepancy between results is smaller than the errors introduced by diseretization for the mesh sizes used and well below differences which eould be observed by plotting.

ln this section some computational results are presented and discussed. The density, pressure and vorticity are sealar functions of the horizontal and vertical coordinates at any specified time. We have found that contours of eonstant value of any of these scalar quantities are a convenient way to display them. Since the temperature and density are inversely related at any particular time, eontours of eonstant density are also contours of eonstant temperature.

All contour plots were preparcd from a graphics package developed by the National Center for Atmospheric Researeh. The numbers indicating contour values are relative only. Solid lines represent values of the variable greater than zero and dashed lines represent values less than zero. For the results presented here, density and temperature are inversely related: eontours of constant density have been labeled as contours of constant temperatures for illustration. Therefore, for the temperature plots, temperature contours above a reference 
value appear dashed and below that reference are solid. Graphic details on the scale of a mesh cell, which is determined by the distance between fiducial marks on the sides of the plots, should be ignored.

All computations have been performed on onc of three computers, the NBS UNIVAC 1108, the U.S. Treasury UNIVAC 1100/81 or the Cybernet Cyber 175. The computations require about 45K words of storage for the $31 \times 31$ mesh and were performed on any one of the three machines. Typical running times on the 1108, which is the machine most frequently used, is about 30 to $45 \mathrm{~min}$ of CPU time for 200 to 300 time steps.

In figure 3 contours of constant temperature (isotherms) are shown at four dimensionless times for a volumetric heat source centcred along the floor in a square room. The rate of heat added per unit volume is largest along the floor at the center of the room and decreases in a Gaussian fashion with horizontal distance from the center and exponentially with height above the floor. The heat source is "turned-on" as a hyperbolic tangent with respect to time asymptoting to full strength around $t=1.0$. (Note that $T$ denotes time on the figure titles.) At the first time, $t=2$, the problem is still linear; the flow velocities are sufficiently small than convection is unimportant, and the temperature increase in the fluid is directly proportional to the volumetric rate of heat added. Therefore, the isotherms are also contours along which the volumetric heataddition rate is constant. (These contours are found to be parabolas.)

These computations were performed on a spatial mesh having 31 cells in the horizontal and 31 cells in the vertical directions; the tick marks along the boundary of the enclosure show the mesh cell spacing.

At time 8.5, the second frame of figure 3, a buoyant thermal has developed, giving the appearance of a mushroom cloud. The buoyant thermal intensifies in strength until the thermal hits the ceiling, as shown in the third frame, $t=11.5$ and begins to spread. Inside the plume a distinctly periodic structure has begun to develop, as can bc seen vividly in this frame; here, progressing up the plume along its centerline, one finds a local low first, then a pcriodic sequence of local highs and lows up to about the center of the head of the thermal.

The heated gases spread along the ceiling and fill the room from the top down, as shown in the last frame of figure 3 at $t=14.5$. This physical behavior is exactly what is observed in room-fire tests and in other experimental observations of heating in enclosures. The symmetry about the centerline of the room displayed in thcsc computations is some measure of the accuracy with which they were performed: the heat sourcc is placed symmetrically, but the computations were performed as if no symmetry existed.

In figure 4 contours of constant vorticity at various times are displayed. The contours show an anti-symmetry about the centcrline, as would be expected for the vorticity, and the physical features described for the density (or temperature) contours are reflected in the vorticity plots. Because the vorticity represents a higher order differcnce of the dependent variables, these contours display more fine scale (on the mesh scale) features than the density contours. Also, later in the computations, "noise" of a nonsymmetric and fine scale begins to show up. The vorticity plots are not as "smooth" as those of the density (or temperature).

In the first frame of figure 4 at time 2.0 , early in the heating process, two vortices of equal magnitude and opposite sign develop with centers in the regions of steepest gradient of the heat source. Convection has begun by dimensionless time $t=8.5$, frame 2 of figure 4 , and the vortices are pinched together and buoyed upward off the floor. The vortices are convected toward the ceiling. Also vorticity of periodically varying strength is generated within the source region and the strength of the vorticity increases with distance above the source. Finally, frames 3 and 4 of figure 4 show that the periodically varying vorticity trains split when the ceiling is encountercd and form two large regions of vorticity of opposite sign.

The pressure as a function of horizontal and vertical coordinates at any specified time can also be displayed. In figure 5 contours of constant pressure at four times during the room filling are shown. This pressure actually represents only the spatial variation of the pressure and has been normalizcd at each time so that its mcan value is zero. This is the quantity which, together with buoyancy, induces the flow. As with the other contour plots, solid lines represent contours with values greater than zero and dashed lines indicate values less than zero. The pressure plots are secn to be smoother than those of density (temperature).

Early, thc first frame at $t=0.5$ of figure 5 , the pressure is highest at the source, where heating takes place. As convection starts, the high pressure region is lifted off the floor, time $t=2.0$ : a significant enough convective velocity has developed by dimensionless time $t=2.0$, that a low pressure region due to a Bermoulli effect can bc seen at the floor. This low pressure region is associated with the high convective velocities, or the vorticity pair shown in figure 4 . The low pressure region develops a double minimum, symmetric about 

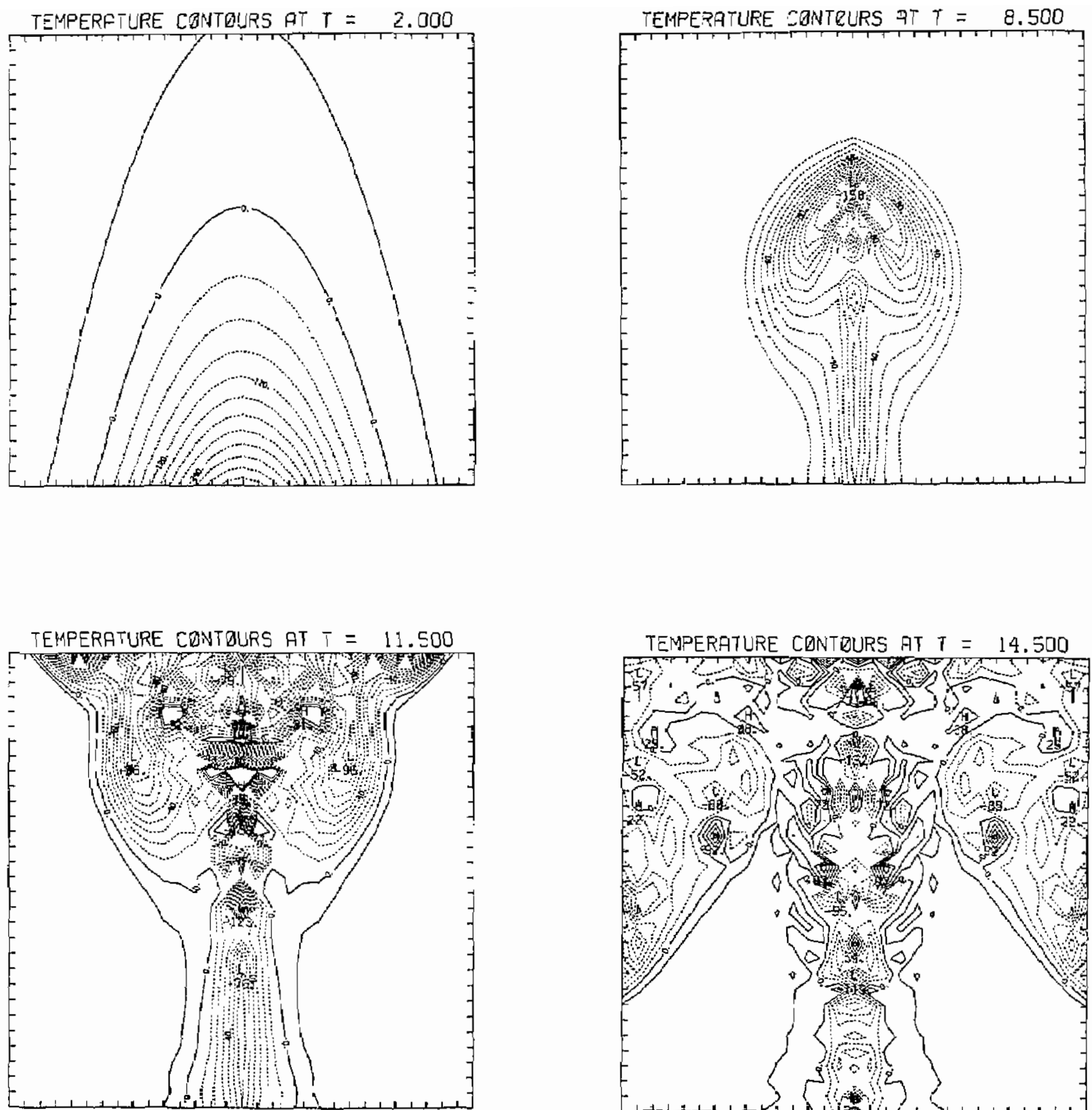

TEMPERATURE CONTOURS AT $T=14.500$

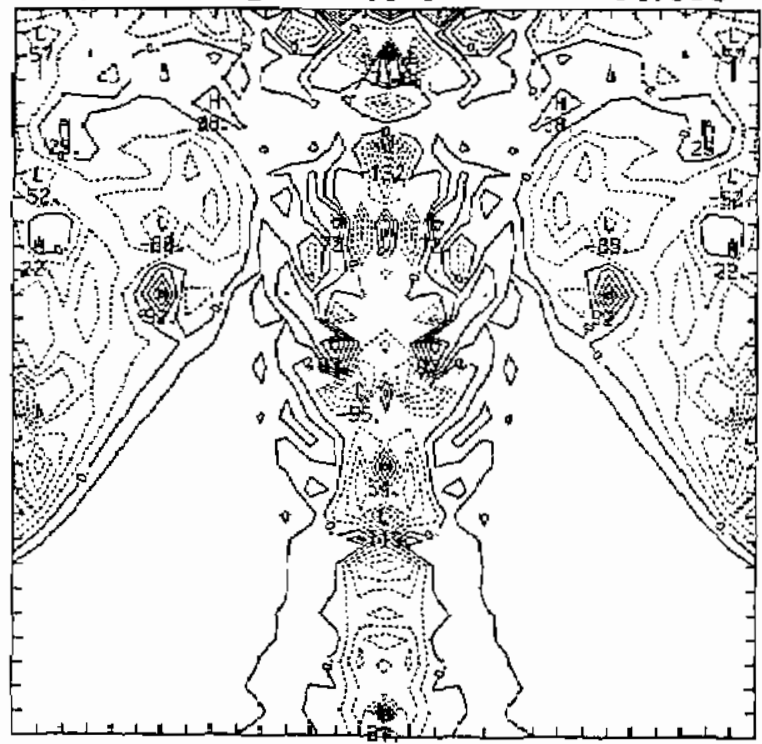

F[GLRE 3. Isothems at four dimensionless times, $T$, during healing by a volumetric heat source centered thlong the floor in a square roon. These four frames illustrate (1) early healing with no convection, (2) a starting plumie, (3) the plume impacting the ceiling, and (4) the room filling from the top doun. 

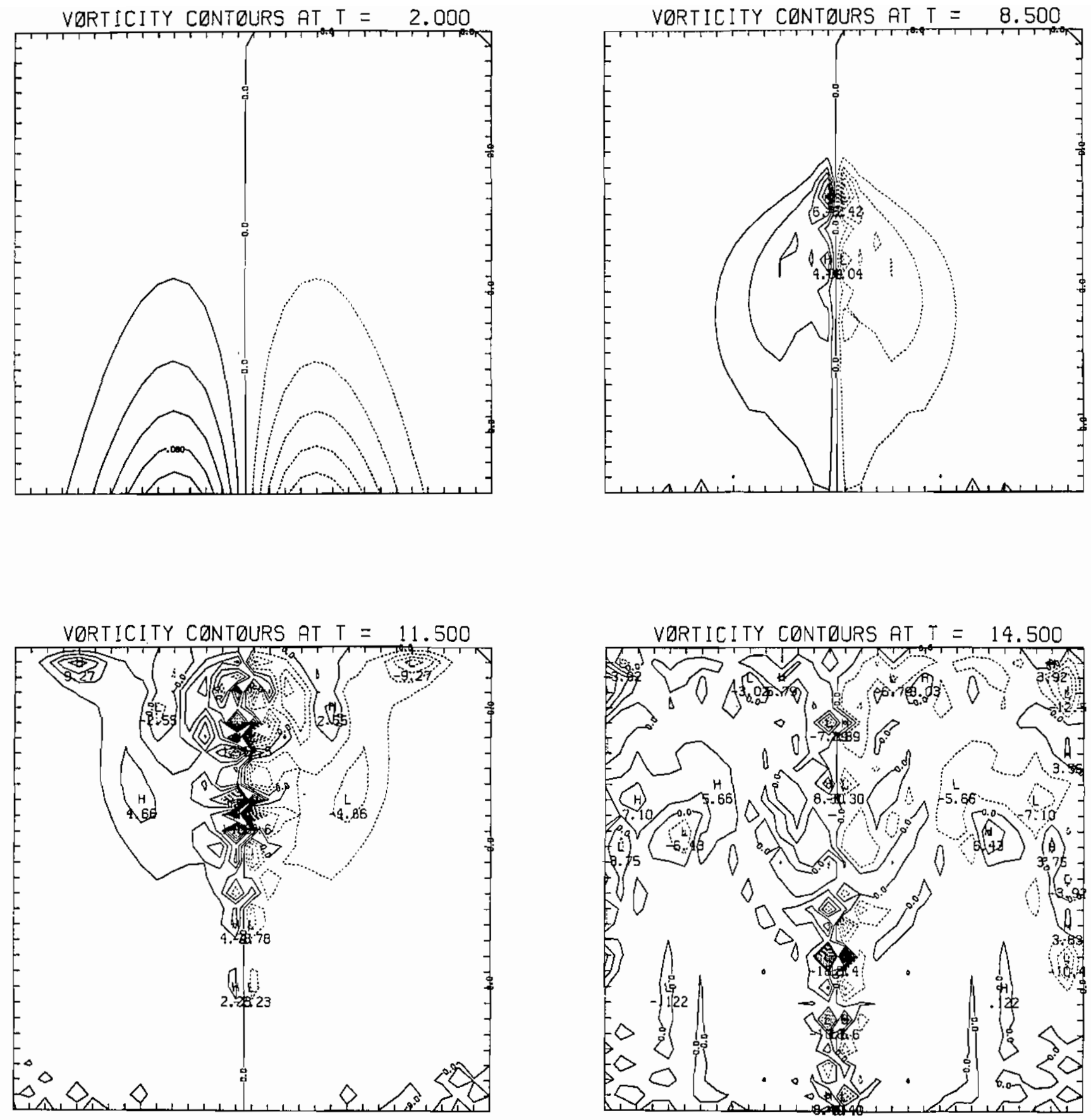

FIGURE 4. Contours of constant vorticity at four dimensionless times, $T$, during heating by a volumelric heat source centcred along the floor in a square room. These four frames correspond to the ones shown in figure 3 . 

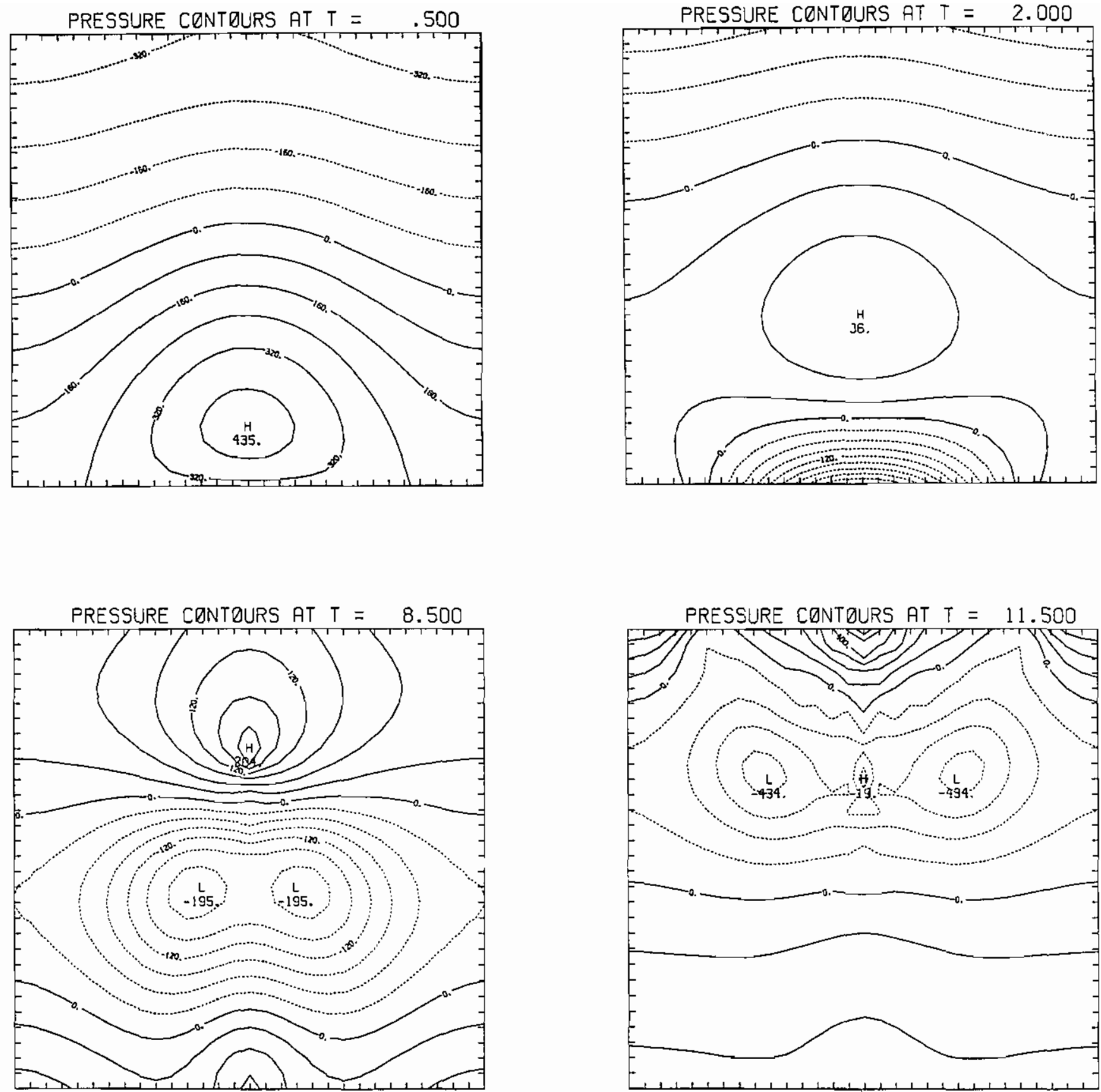

FIGURE 5. Contours of constant pressure at four dimensionless times, $T$, during heating. The calculation shown herc is the same as that displayed in figures 3 and 4 , but the times are chosen to illustrate the stages in the development of the pressure ficld. 
the room centerline as seen at time 8.5 and rises to the cciling, as shown in figure 5 , time 11.5 , where a strong compression develops at the center of the ceiling. The final prcssure diagram displays the double minimum, associated with the strong vortex pair and high temperature shown in figurcs 3 and 4 , scparating and moving toward the walls.

In figures 6 and 7 contours of constant potential $\phi$ and constant stream funetion $\psi$ are shown. Only one plot of the potential is shown because the spatial dependence does not change with time: the potential function is separable in space and time. Four frames of stream function are shown in figure 7. The stream function is antisymmetrical about the centerline and displays a peak and a valley which slowly rise toward the ceiling. The stream function is rather smooth. showing only a slightly wavy behavior at later times.

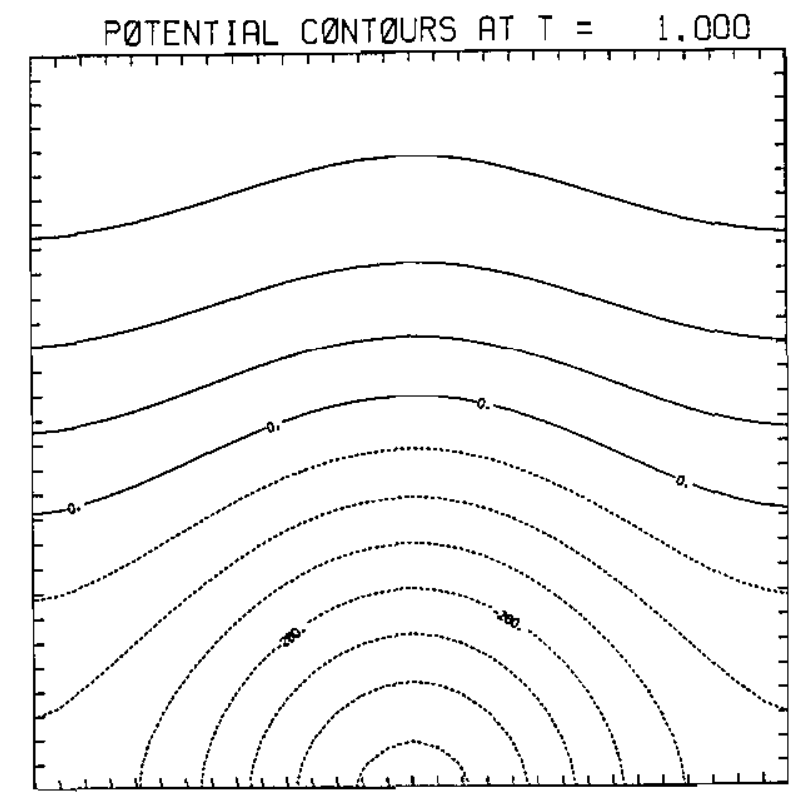

FigurE 6. Velocity-potential conlours al dimensionless time, $T=1.0$ for the calculation displayed in figures 3-5. The potential function is a function of space times a function of time; therefore, the spalial dependence does not change with time.

The base computation, shown in figures $3-7$, was repeated several times with smoothing introduced at diffcrent numbers of time steps. Figures 8 and 9 compare constant temperature contours at dimensionless time 11.5 and 14.5 respeetively determined by the base eomputation, on the upper left, and by three levels of smoothing in the other three plots of each figure. It is seen that the fine structure is eliminated by smoothing, but large scale features are still retained. In figure 8 four plots of constant temperature contours at approximately the same time are compared. The plot in the upper left hand corner is the unsmoothed computation. The plot at the upper right is the result of the computation smoothed only once up until that time, after $N=160$ time steps. The next plot, lower left, is from a computation smoothed every 80 time steps, that in the lower right is from a computation smoothed every 40 time stcps. Figure 9 shows a similar comparison of the effects of smoothing, but at approximately $t=14.5$. These plots show clearly the loss in detail with increasing frequency of smoothing or inereased simulated viscosity. They also show that the buoyant plume rise slows due to the decreasing gradients. The smoothing and loss of fine-scale detail with incrcased frequency of smoothing are apparent, and, in fact, these results appear much closer qualitatively to results obtained in previous studies which integrated the Navier-Stokes equations by finite difference techniques (for example, reference [9], figures 4 and 5). 

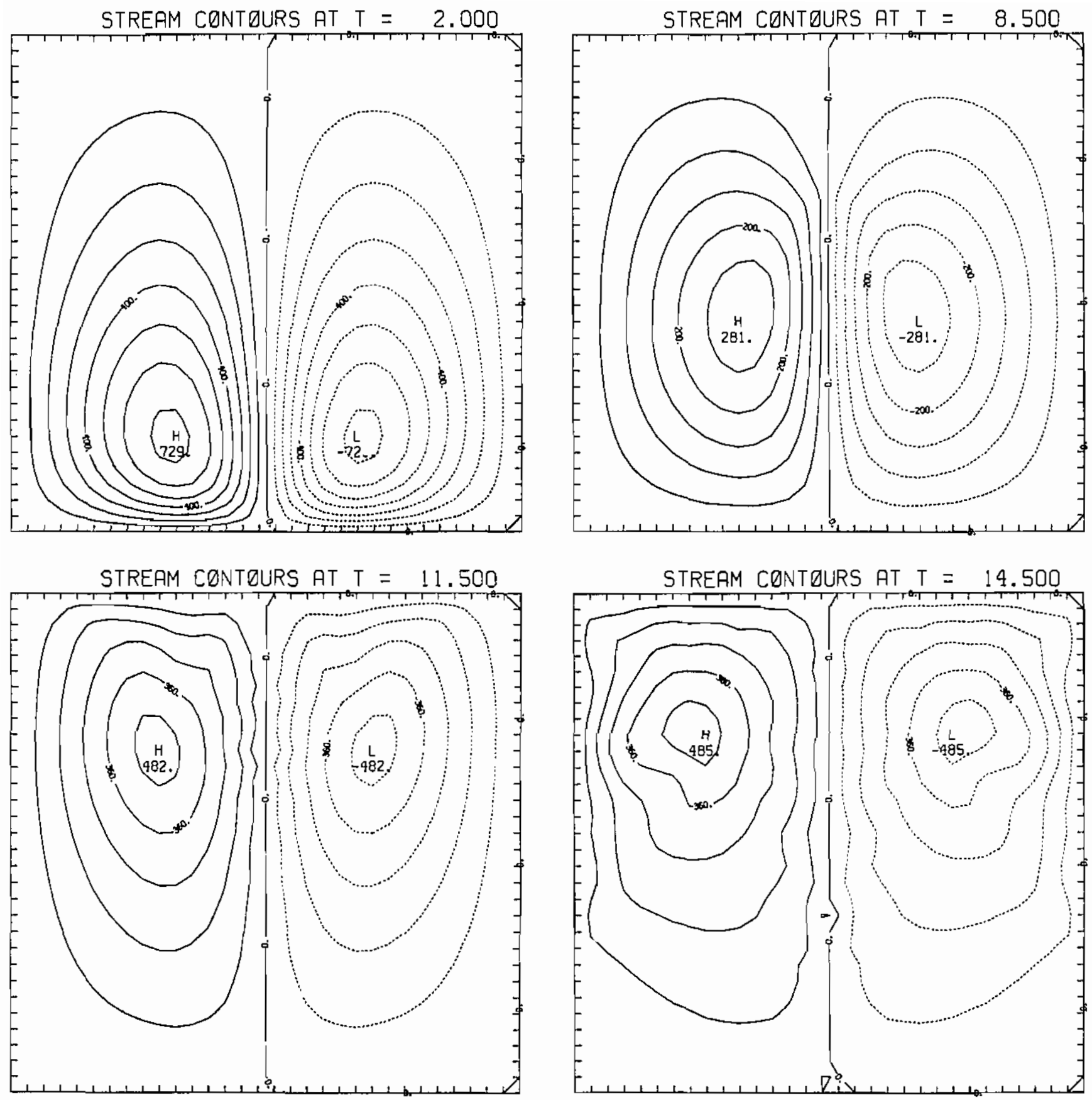

Figure 7. Stream-function contours at four dimensionless times corresponding to the limes displayed in figures 3 and 4. 

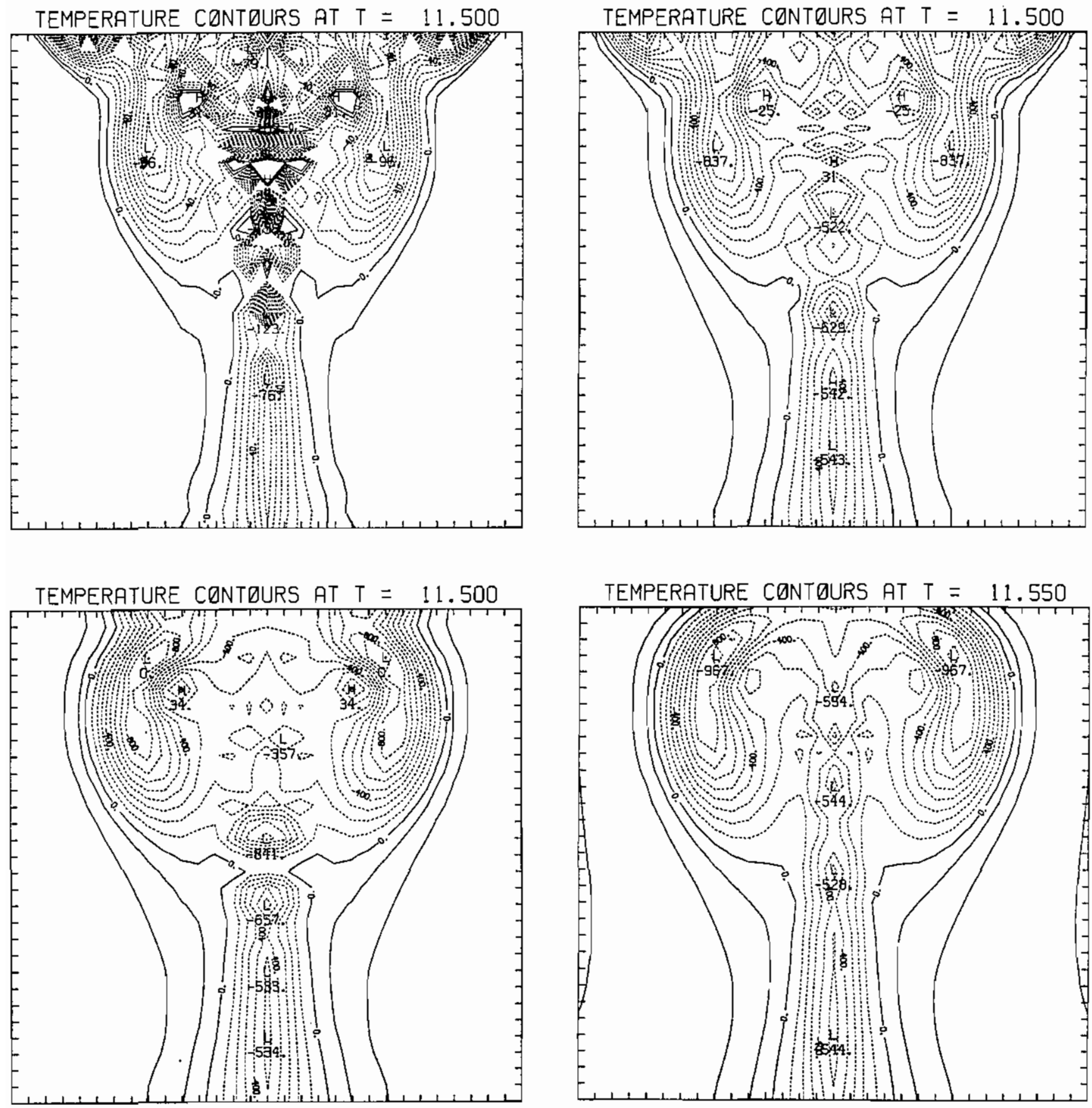

FIGURE 8. Isotherms at approxinately the same dimensionless time, $T$, with Lanczos smoothing applied al different frequencies. The frame in the upper left comer is the unsmoothed computation. The frame at the upper right is smoothed every 160 time steps, thal at the lower left every 80 time steps and that at the lower right every 40 time steps. 

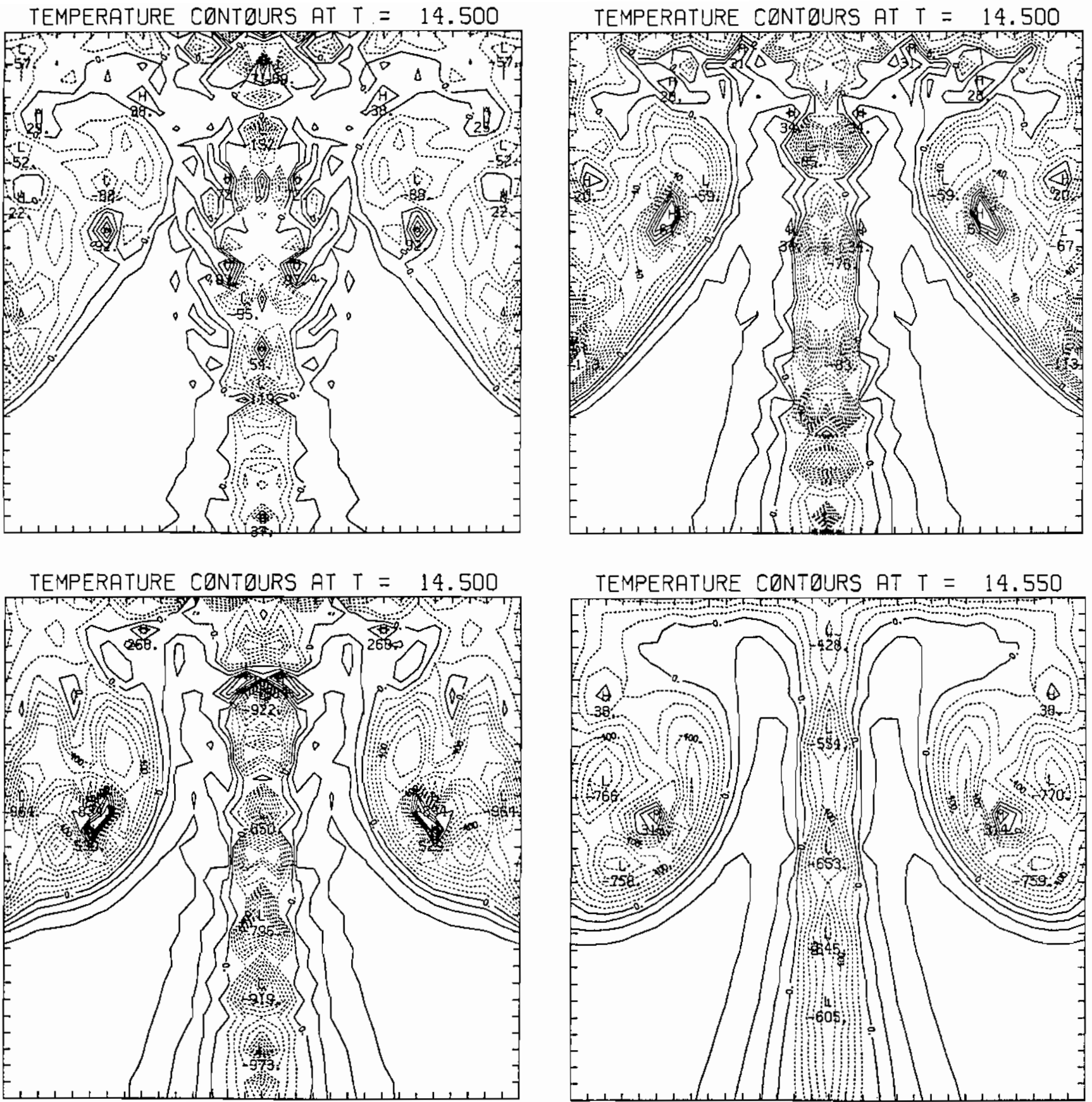

Figune 9. Isolherms at approximately the same dimensionless time, $T$, with Lanezos smoothing applied at different freqnencies. The frame in the upper left eorner is the unsmoothed computation. The frame in the upper right is smoothed every 160 time steps, thal at the lower left every 80 timc steps and that at the lower right every 40 time steps. 


\section{References}

[1] Rehm, R. G. and Baum, H. R., The Equations of Molion for Thernally Driven Buoyant Flows, J. Res. Nat. Bur. Standards 83, No. 3, pp. 297-308 (May-June 1978).

[2] Baum, H. R., Rehm, R. G., Barnett, P. D., and Corley, D. M., Finite Difference Calculations of Buoyant Convection in an Enclosure, Part I: Basic Algorithm, Nal. Bur. Stand. (U.S.) NBSIR 81-2385 (Dec. 1981).

[3] Rehm, R. G., Baum, H. R., Barnelt, P. D. and Corley, D. M., Finite Difference Calculations of Buoyant Convection in an Enclosure, Part II: Verifieation of the Basie Algorithm, National Bureau of Standards Technical Nole in preparalion.

[4] Baum, H. R. and Rehm, R. G., Finite Difference Solutions for Intemal Waves in an Enclosure, National Bureau of Standards Report in preparation.

[5] Harlow, F. H. and Amsden, A. A., Fluid Dynamics, A LASL Monogroph, Los Alamos Scientific Laboratory Report LA4700, Los Alamos, New Mexico (June 1971).

[6] Swarzlrauber, P. and Sweet, R., Efficient FORTRAN Subprograms for the Solution of Elliptic Partial Differenlial Equations, NCARTN/IA-109 (July 1975).

[7] Lewis, J. G. and Rehm, R. G., The Numerical Solution of a Nonseparable Elliptic Partial Differential Equation by Preeonditioned Conjugate Gradients, J. Res. Nat. Bur. Staudards 85, No. 5, pp. 367-390 (Sept.-Oct. 1980).

[8] Lanczos, C., Applied Analysis, Prentice Hall, Inc., Englewood Cliffs, New Jersey, 1956, pp. 316-320.

[9] Knight, C., Numerieal Studies of Natural Conveetion in an Enclosure, Harvard Universily Division of Engineering and Applied Physies, Technieal Report No. 15.

[10] Kreiss, H. and Oliger, J., Methods ior the Approximate Solution of Time Dependenl Problems, Global Atmospheric Research Programme (GARP) Pnbliealion Series No. 10, February 1973. 